\title{
LA CREACIÓN PUJA Y DIOS CON ELLA: ESTUDIO DE LA ARGUMENTACIÓN EN ROM 8,18-30
}

\author{
Creation pushes and God with it: study of the argumentation in Rom 8,18-30
}

\author{
Juan Manuel Granados ${ }^{1}$
}

\section{Resumen}

Este artículo propone el estudio exegético de Rom 8,20-21 y busca responder a la pregunta: ¿quién” sometió la creación al fracaso? La discusión exegética a propósito del significado de la expresión "por medio de aquél

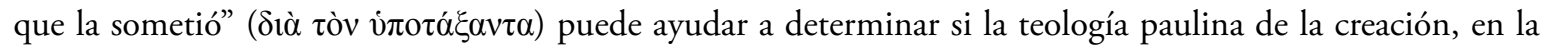
carta a los Romanos, se debe entender en términos escatológicos o más bien cristológicos. La afirmación de la liberación de la creación en Rom 8,21 también indaga por el tipo de corrupción del cual el texto trata. ¿Por qué y para qué la creación fue sometida "en esperanza"? Esta investigación ofrece un enfoque retórico a Rom 8,18-30 y muestra el crescendo (auxesis) de los gemidos: la creación gime, nosotros gemimos, el Espíritu gime. Este crescendo incluye la comprensión que tienen los creyentes de la acción divina por medio de Cristo. Pablo usa, en este texto, la figura retórica de la "personificación" (prosopopeya) para identificar la creación humana y sub-humana. Pablo también relaciona esta figura con otra, la figura etimológica o juego de palabras, a partir de la raíz gramatical del "gemir"; de esta manera, muestra una paradoja: en Cristo, el sufrimiento humano y el "pujar" de Dios suceden de forma conjunta. Se concluye así que, en esta parte de la carta, Pablo demuestra que en Cristo tienen lugar no sólo la nueva creación, sino también la conciencia humana de ser hijos de Dios.

Palabras clave: Rom 8,18-30; Análisis Retórico; Prosopopeya; Incrementum; Filiación Divina; Teología de la Creación; Nueva Creación.

1 Doctor en Ciencias Bíblicas y Licenciado en Sagrada Escritura, Pontificio Instituto Bíblico (PIB), Roma, Italia. Profesor Asociado Facultad Bíblica -PIB. Correo electrónico: jmgranados@biblico.it 


\section{Abstract}

This paper puts forward an exegetical study of Rom 8:20-21 and attempts to explain "who it was that subjected creation to failure." The exegetical discussion regarding the meaning of the expression "by the will of the one who subjected it" ( $\delta$ ì̀ $\tau o ̀ v ~ v i \pi o \tau \dot{\alpha} \xi \alpha v \tau \alpha$ ) helps to determine whether the Pauline Theology of Creation in Romans is to be interpreted as eschatological or Christological. The announcement of the liberation of creation in Rom 8:21 also raises the issue of "its bondage to decay." Why and for what purpose was creation subjected "in hope"? This research proposes a rhetorical approach to Rom 8:18-30 and reveals a crescendo (auxesis) in the groaning: the creation groans, we groan, and the Spirit groans. This crescendo involves the believers' understanding of the effects of God's salvific action through Christ. Paul uses the rhetorical figure of "personification" (prosopopeia) to identify human and non-human creation. Paul also relates this figure of thought with a play on words (groans / groaning) to show a paradox: in Christ, human suffering and God's help to give birth to new life come together. Thus, Paul intends to demonstrate not only a "New Creation" in Christ, but also a new awareness: that of divine filiation.

Keywords: Rom 8:18-30; Rhetorical Analysis; Prosopopeia; Incrementum; Divine Filiation; Theology of Creation; New Creation.

\section{Introducción}

¿Por qué afirma Pablo que "la creación fue sometida al fracaso por aquel que la sometió" (Rom 8,20)? ¿Y cómo la sometió? Las traducciones ubican alternativamente los complementos adverbiales: "no por su propia voluntad", sino "con—o en—esperanza”. Las dos dificultades más importantes que presenta la

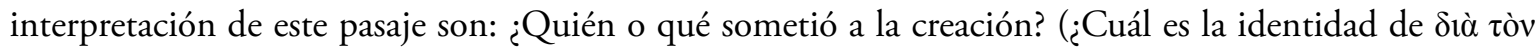

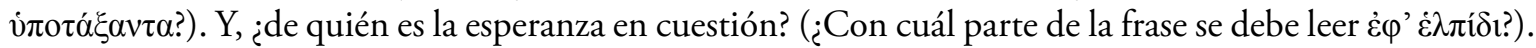
Los comentarios más recientes no logran ponerse de acuerdo, tampoco las traducciones. Jewett sugiere que la frase podría aludir al Génesis. Pablo habría personificado la futilidad del pecado causado por Adán, Eva y sus descendientes. Por esta misma razón, se debe precisar que la creación no fue sometida por su propia culpa, sino involuntariamente ${ }^{2}$. Käsemman (1974) afirma que el participio debería corresponder al mismo sujeto, a saber, Dios (p. 227), mientras que Wilckens (1978) se inclina por Cristo (p. 154). Dunn (1988) señala la posible incoherencia de la hipótesis que adjudica a Adán tal culpa, "in that case we would expect a reference to Adam's failure, not to his God-given function on account of Adam's transgression" (p. 471), y se decanta, finalmente, por Dios. Los especialistas de la carta no han encontrado, hasta ahora, un acuerdo razonable que explique este crux interpretum; la mayoría de ellos concentrados, solamente, en

2 La misma idea se encuentra en el Apocalipsis de Esdras: "Propter eos enim feci saeculum, et quando transgressus est Adam constitutiones meas, iudicatum est quod factum est" (VUL). "Cuando Adán transgredió mis preceptos, cuanto había sido hecho fue juzgado” (4 Esdras 7,11). (Cf. Jewett y Kotansky, 2007, p. 514). 
el vocabulario o en el contexto bíblico de la afirmación. Otra solución se abre camino, lentamente, entre los estudiosos que analizan la argumentación paulina con las herramientas de la retórica antigua.

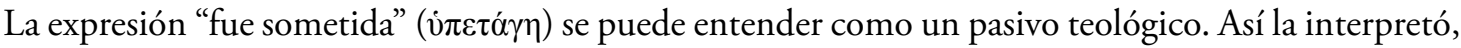
por ejemplo, Juan Crisóstomo en sus homilías a la carta.

¿Qué significa: la creación fue sometida a la frustración? Que ha llegado a ser corruptible. ¿A causa de quién y por cuál razón? Por tu causa, hombre. Porque amén de que [tu] hayas recibido un cuerpo mortal y expuesto al sufrimiento, también la creación recibió una maldición, y lleva consigo cardos y espinas. Sin embargo, el cielo caduco junto con la tierra, más tarde se transformará en una porción-lote de heredad-mejor. ( In Espistulam ad Romanos, 60.530.3-8). ${ }^{3}$

Crisóstomo prueba enseguida su argumento de la "transformación" ( $\mu \varepsilon \theta i ́ \sigma \tau \eta \mu \mathrm{l})$ por medio del Sal 101,27-28 (LXX) y de Is 51,6; como un vestido viejo y gastado se cambia y transforma, así, también, la creación (Schaff, 1974-75, p. 597). Esta opción, sin embargo, ha suscitado un impase para la, así llamada, teología de la creación, pues ella parece estar en contradicción con la descripción de una creación buena

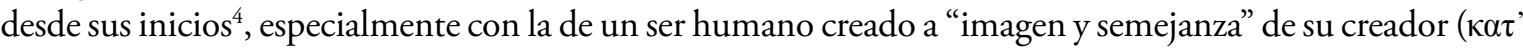

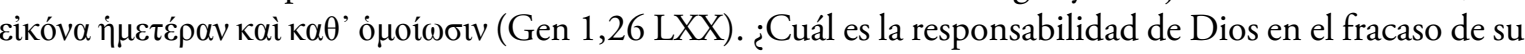
propia creación? ¿Tiene el ser humano la "culpa" de tal fracaso? Algunos teólogos creen que preguntarse por las responsabilidades no tiene mucho sentido y, en su lugar, buscan el propósito de la frustración, tanto la humana como la de la creación. Es muy posible que la teología de la creación propuesta por Pablo en la carta a los Romanos tenga características escatológicas y se entienda solo a la luz de la esperanza y de la gloria futura. Esta interpretación escatológica puede ser plausible, pero puede que no dé razón suficiente de los sufrimientos presentes de la creación.

A continuación, se sintetizan los resultados del estudio de la argumentación paulina en Rom 8,18-32. Esta investigación busca demostrar que el apóstol articula diversas figuras retóricas, a saber: prosopopeya ( sufrimientos presentes no pueden agotar la esperanza en una salvación definitiva. La demostración paulina, en esta sección, tiene la forma de un crescendo que va del exterior hacia el interior. El hilo del pensamiento es temporal y se expone alternando el pasado de cuanto Cristo ha hecho por los creyentes, el futuro que les cabe esperar y las consecuencias para las dificultades que tienen que afrontar en el presente. La conclusión más importante que se puede anticipar de este estudio atañe a la teología paulina de la creación en la carta a los Romanos, a saber: la creación participa de los mismos efectos de la redención destinados a los hijos de Dios. Los hijos-y toda la creación con ellos-dan gloria al Padre y son su gloria. La forma de

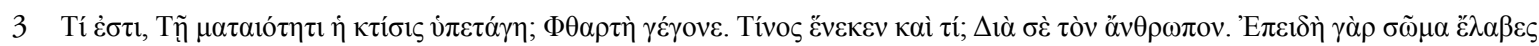

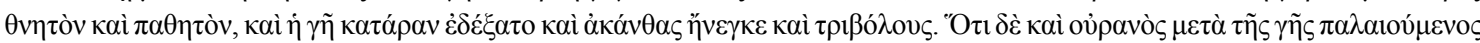

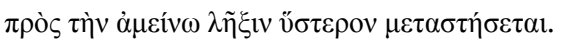

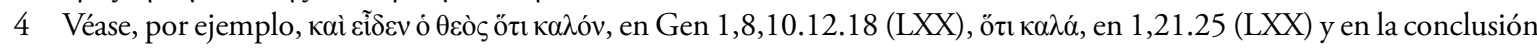

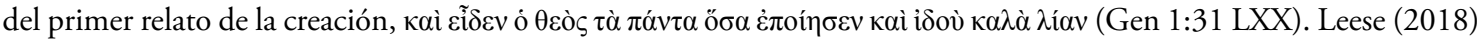
afirma, por ejemplo, que en las cartas paulinas, en pasajes como Rom 8,18-30, el apóstol usa el mismo campo semántico y conceptual de Gen 1,26-27 y 2,7 (p. 127). 
argumentar del apóstol persigue, no solo informar a sus destinatarios de los beneficios que les esperan si creen en Cristo, sino cambiar su manera de pensar ( $\varphi$ óv $\eta \mu \alpha)$, de tal manera que puedan modificar sus expectativas. Para quienes tienen las primicias del Espíritu, la pregunta por los padecimientos presentes no es por qué, sino para qué. La presentación que sigue se desarrolla en tres partes principales: observaciones semánticas sobre el vocabulario del gemir ( $\sigma \tau \varepsilon v a ́ \zeta \omega)$; explicación del incrementum en la argumentación de la sección y una breve reflexión teológica sobre la salvación y la creación.

\section{El vocabulario otعvá̧ $\omega$ y sus compuestos}

La repetición de los términos compuestos con la raíz $\sigma \tau \varepsilon v-$ se podría interpretar como un ejemplo más de la maestría paulina para utilizar el lenguaje. Sin embargo, una lectura detenida sugiere que esta repetición obedece a razones argumentativas. La repetición de términos corresponde a una figura retórica conocida por Pablo. La modulación de un mismo término con significados diferentes se denomina políptoton (Granados, 2002, pp. 153-170). Los rétores antiguos apenas distinguían esta figura de la paronomasia o semejanza fonética entre vocablos. ${ }^{5}$ Estudiosos más recientes han distinguido entre la modulación nominal (también preposicional) y la modulación de raíces verbales. La primera, ya conocida como políptoton, la segunda, como figura etimológica $(\pi \alpha \rho \eta \gamma \mu \varepsilon ́ v o v){ }^{6}$ ¿Cuál es la figura que describe mejor la repetición de la raíz $\sigma \tau \varepsilon v-$ ? Quizá, más importante, ¿con qué objetivo la utiliza el apóstol en esta sección??

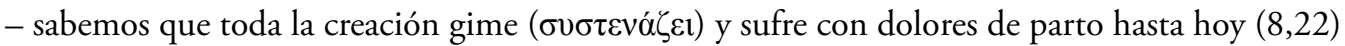

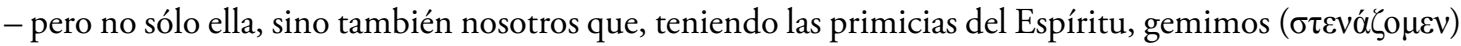
en nosotros mismos esperando la filiación, la redención de nuestros cuerpos $(8,23)$

- de igual forma, el espíritu nos ayuda en nuestra debilidad, pues no sabemos pedir como se debe; sin embargo, el espíritu mismo intercede con gemidos inefables ( $\sigma \tau \varepsilon v \alpha \gamma \mu$ oĩ $\grave{\alpha} \lambda \alpha \lambda \eta \dot{\tau o l \varsigma)}(8,26)$.

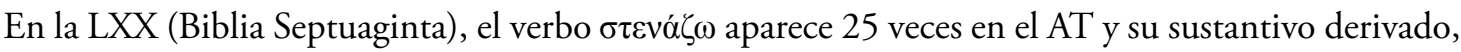
$\sigma \tau \varepsilon v \alpha \gamma \mu$ ó, 28 veces. El verbo está asociado generalmente a expresiones de dolor y llanto, ${ }^{8}$ a experiencias

5 "Tertium est genus figurarum quod aut similitudine aliqua vocum aut paribus aut contrariis convertit in se aures et animos excitat. Hinc est $\pi \alpha \rho o v o \mu \alpha \sigma i \alpha$, quae dicitur adnominatio. Ea non uno modo fieri solet”. (Quintiliano, Inst., 9.3 [Russell, LCL]).

6 Lausberg (1998) define la paronomasia como un juego de palabras pseudo-etimológico que estimula al oyente para descifrar el significado creado por el cambio de orden en las palabras (\$ 637). Mortara Garavelli (1988) distingue entre las figuras de repetición que varían su forma la paronomasia, la políptoton y la figura etimológica $(\pi \alpha \rho \eta \gamma \mu \varepsilon ́ v o v$ o derivatio). Su comprensión de la última permite comprender mejor la derivación de términos que abarca no sólo nombres, sino todas las formas de un sema (186. 206-210). Véase también http://rhetoric.byu.edu/ (ad loc.).

7 La diferencia sutil entre paronomasia y figura etimológica no altera sustancialmente nuestro análisis de los compuestos con la raíz $\sigma \tau \varepsilon v-$ (si bien el autor se inclina por la segunda). La verdadera importancia de la repetición emerge, con claridad, cuando se evidencian sus sujetos y la secuencia que ellos conforman: creación, nosotros, Espíritu.

8 Véase Is 19,18; Ez 21,11-12; 26, 16 (similar al pánico); Tob 3,1; Job 30,25; 31,38; Sab 5,3; eventualmente, como asombro (Job 18,20). 
de decaimiento, ${ }^{9}$ a la opresión, ${ }^{10}$ y ocasionalmente asociado al arrepentimiento. ${ }^{11}$ La mención del gemir de la tierra "y todos sus surcos que se llenan de llanto" (Job 31,38) podría indicar el contexto sapiencial de la afirmación. Sin embargo, el análisis del sustantivo derivado propone otro contexto posible para la interpretación de este gemir. El gemido aparece asociado, igualmente, al llanto y al dolor, ${ }^{12}$ al lamento y a la queja, ${ }^{13}$ al decaimiento o desfallecimiento, ${ }^{14}$ o para describir el efecto de la opresión y la persecución. ${ }^{15}$ La expresión "como gemidos de parturienta" en Jr 4,31, al igual que las palabras del castigo divino en Gn 3,16, ${ }^{16}$ reflejan mejor el contexto de Rom 8,22. Si bien el verbo $\sigma \tau \varepsilon v \alpha ́ \zeta \omega$ y el sustantivo derivado, $\sigma \tau \varepsilon v \alpha \gamma \mu$ ós, podrían aludir al dolor causado por un agente externo, fruto de la opresión, o a experiencias de sometimiento violento, la imagen de la mujer parturienta, así como la evocación prototípica de Eva, sugieren un contexto ligeramente diferente. El gemir y los gemidos en cuestión se parecen más a un empujar de adentro hacia afuera, como el de una mujer que da a luz sus hijos pujando con dolor.

En la literatura extrabíblica $\sigma \tau \varepsilon v \alpha ́ \zeta \omega$ y $\sigma \tau \varepsilon v \alpha \gamma \mu o ́ \varsigma$ significan también suspiro profundo o gemido (Schneider, TDNT 7:600-603). Filón de Alejandría, por ejemplo, refiriéndose a Gn 3,16, utiliza el

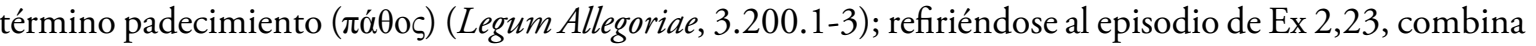

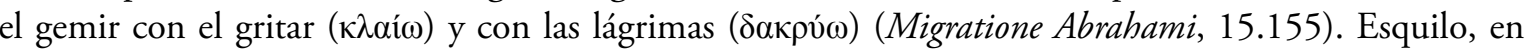
Los Persas, utiliza la expresión en paralelo con los gritos de dolor por Atenas: "Ay, ¡cuántos lamentos me causa el recuerdo de Atenas!” 17 Su narración del combate contra una barca fenicia muestra que el verbo también formaba parte de la jerga militar para indicar "apelotonarse" o "hacer presión". ${ }^{18}$ Los ejemplos citados confirman que el uso paulino de la expresión es singular. La semántica de $\sigma \tau \varepsilon v-$ abarca un sinfín de significados relacionados con el gemir y sus diversas causas. Sin embargo, el apóstol circunscribió su significado al dolor que emerge con fuerza cuando se genera la vida. Este uso peculiar sobresale aún más

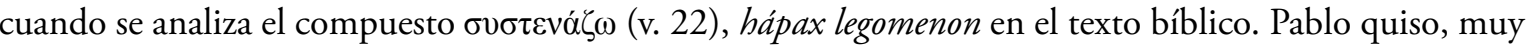
posiblemente, jugar con la sonoridad de la raíz, pero más importante que ello, la utilizó de tal forma que subrayó su contenido "generador".

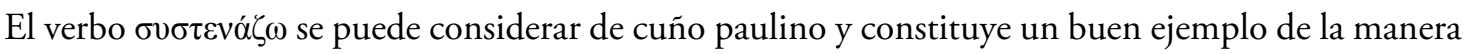

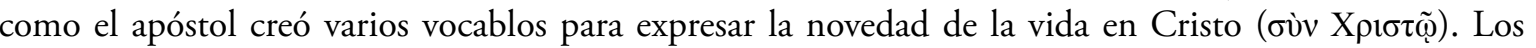
compuestos $\sigma v v$ - sugieren no sólo cierta creatividad y flexibilidad en el uso del lenguaje, sino también cierto "forzamiento" en su manejo. Los ejemplos más representativos en la carta a los Romanos se concentran

9 Véase Is 59,10 (gemir como un muerto); Ez 26,15 (gemir del herido); Job 24,12; 1 Mac 1,26.

10 Relacionado también con amenazas externas, véase Is 21,2; 30,15; 4 Mac 9,21.

11 Véase Is 46,8; Jr 38,19; Lam 1,8. En Nah 3,7 significa, además, lamentarse (דונ), como hacer duelo por alguien o sentir compasión. En Mc 7,34 se entiende como simple "suspirar".

12 Véase Gn 3,16; Is 35,10; 51,11; Jr 45, 3; Ez 24,17 (como luto); Mal 2,13; Sal 6,7; Job 3,24; $23,2$.

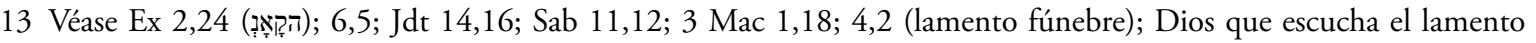
humano: Hch 7,34.

14 Véase Sal 31,11; 38,9; 101,6; Lam 1,22.

15 Véase Jue 2,18; Sal 11,6; 79,11; 102,21; Salmos de Salomón 4,14. El gemido aparece, en fin, como suspiro en la oración (Tob 3,11) o como suspiro de ansiedad (Sal 37,10).

16 "Multiplicaré tus dolores en el embarazo y darás a luz con gemidos de dolor" (Gn 3,16a).

17 Véase $\tau \tilde{\omega} v$ A $\theta \eta \nu \tilde{\nu} \omega \dot{\omega} \varsigma \tau \varepsilon \dot{v} \omega \mu \varepsilon \mu \nu \eta \mu \varepsilon ́ v o \varsigma$ (Esquilo, Los Persas, [traductor Fernández Galiano], p. 230).

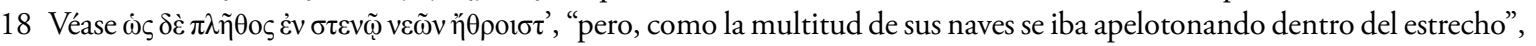

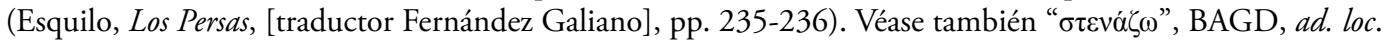




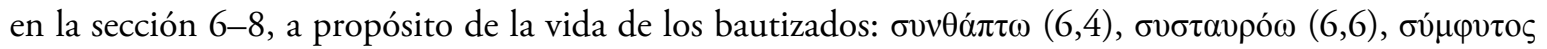

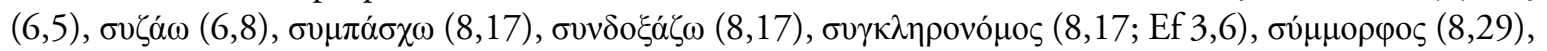

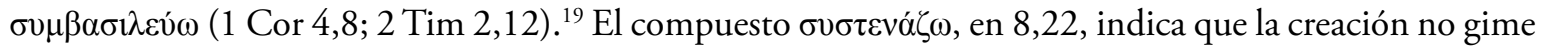
sola. Solo gracias a la técnica de la repetición, el lector puede comprender que ella gime junto con nosotros y junto con el Espíritu. Leese (2018) reconoce, por ejemplo, que: "Paul correlates the groaning of creation with groaning of humanity" (p. 114) y está de acuerdo con Jewett (2007) cuando afirma que: "God's Spirit dwells 'among,' 'within,' or 'in the midst of' ('่v) the congregation, rather than merely within the heart of individuals" (p. 489). El análisis de los compuestos $\sigma \tau \varepsilon v-$ sugiere, además, que este gemir no describe un sufrimiento pasivo, sino la expectativa de una vida que comienza.

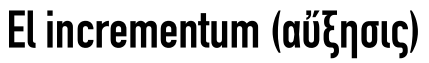

¿Por qué la creación fue sometida a la frustración "contra su voluntad"? La calificación adverbial

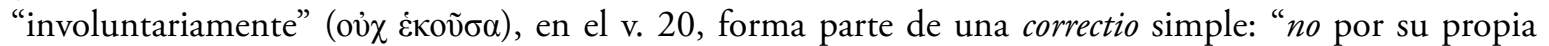
voluntad sino a causa de quien la sometió". El participio atributivo sustantivado "el que la sometió" (

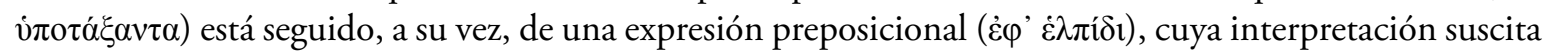
gran debate: ¿Cómo se debe interpretar la sintaxis de la frase?, ¿Cuál es la creación en cuestión?

La sintaxis del v. 20 puede corroborar el tipo de sometimiento, no sólo involuntario, sino también expectante de la creación. La frase preposicional $\dot{\varepsilon} \varphi{ }^{\prime} \dot{\varepsilon} \lambda \pi^{i} \delta \imath$ puede funcionar como un calificativo de tòv

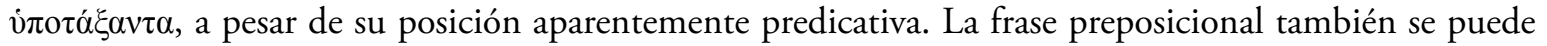

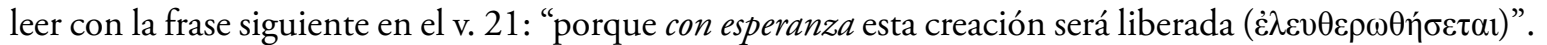
En el primer caso se subraya el sujeto agente que la sometió con esperanza, en el segundo, en cambio, se subraya la creación misma que será liberada con esperanza o que espera la liberación. Esta esperanza reaparece en el v. 24 como ya cumplida: "porque con esperanza fuimos salvados". La dificultad exegética, a propósito de las frases preposicionales y del dativo ( $\dot{\varepsilon} \varphi ’$ $\left.\dot{\lambda} \lambda \pi^{i} \delta \mathrm{r}\right)$, se relaciona así con otra de tenor más hermenéutico: ¿Qué tipo de esperanza tiene Pablo aquí en mente?: spes qua o spes quae?

La Carta Encíclica Spe Salv,i de Benedicto XVI (2007), abordó la tensión hermenéutica entre el contenido de la esperanza y la actitud con la cual se espera. El testimonio de la carta a los Romanos previene contra el solipsismo: a saber, contra un modelo de esperanza individualista encerrada en formas sutiles de voluntarismo y promueve, por el contrario, una visión teleológica del esperar humano: "el presente está marcado por la realidad futura, y así las realidades futuras repercuten en las presentes y las presentes en las futuras" (Spe Salvi, 7). ${ }^{20}$

La segunda dificultad hermenéutica que aparece en los vv. 20-21 concierne a su contenido. ¿̇ncluye esta creación solo las criaturas sin culpa-creación subhumana-o también a las criaturas conscientesresponsables - del propósito divino? Lo estudiosos, también en este caso, se decantan a favor de una u

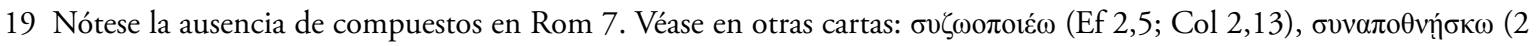

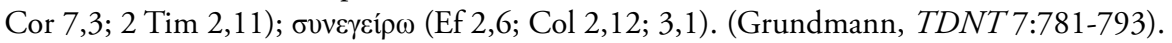

20 Véase en particular "la verdadera fisonomía de la esperanza" (\$\$ 24-31). 
otra interpretación. Sin embargo, el procedimiento literario ya insinuado de la personificación sugiere que Pablo entiende por creación todas las criaturas, tanto las conscientes como las inconscientes. ${ }^{21}$ ¿Por qué habría sometido la creación? ¿Se ha de suponer que ella desobedeció y que la sujeción forma parte del proceso necesario para disciplinarla? ¿Por qué-o cómo se explica, entonces,-que tales criaturas se vean expuestas al sufrimiento o a otras formas de opresión? ${ }^{22} \mathrm{El}$ mismo interrogante ya aparece insinuado en el episodio mencionado en Gn 3,16. Veamos porqué.

El desarrollo del razonamiento en los vv. 18-30 está enmarcado por el motivo de la gloria: la gloria que

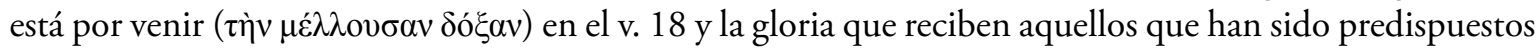

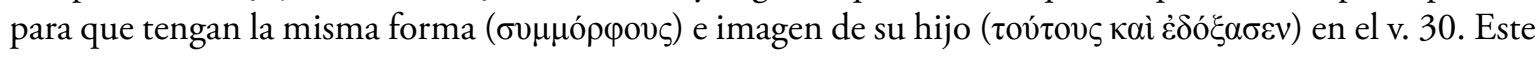
motivo-que constituye una inclusio — no abarca solamente a los hijos, sino también a la creación. La progresión que se insinúa aquí va de la gloria que se manifestará en los hijos a la gloria que Dios les concede. La gloria constituye, así, una expectativa de futuro de los creyentes y de la creación. El crescendo final (vv. 30) subraya, sin embargo, que la expectativa ya se ha cumplido. ¿Cómo se entiende tal cumplimiento? Y, sobre todo, ¿`cómo se explica que los sufrimientos presentes no frustren ni la gloria futura ni la venidera?

(A) La gloria que está por venir (v. 18):

- la gloria que está por manifestarse en nosotros

- el anhelo de la creación que aguarda la revelación de los hijos de Dios

(B) La creación gime con y sufre hasta hoy (aguarda la libertad de la gloria de los hijos de Dios)

(C) Nosotros gemimos (esperando la filiación)

(B’) El Espíritu intercede con gemidos inefables (viene a ayudar nuestra debilidad)

(A') La gloria de los hijos de Dios

- A quienes llamó y justificó, a estos los glorificó (v. 30).

La semántica de la gloria se entreteje con la de la filiación y la de la progenie. De esta forma, Pablo subraya, al final de la sección 5-8, que el destino de los creyentes, justificados por la fe, es el mismo que el trazado para Abraham y sus descendientes $(4,20 \mathrm{cf} .8,17) \cdot{ }^{23}$ Esta progresión muestra, también, que la secuencia del gemir está entrelazada al motivo de la filiación y de la progenie; ella confirma que el gemir está asociado a un pujar, o a un esperar de forma activa el advenimiento de una nueva realidad, a saber,

21 Gieniusz, A. (1999) confirma la lógica de la inclusión, subrayando que esta "gives full value to the phrase $\pi \tilde{\alpha} \sigma \alpha \dot{\eta} \kappa \tau i ́ \sigma ı \varsigma$ in v. 22 which otherwise has to be [...] reduced to only a part of creation, namely subhuman" (p. 194). Stenschke (2017) sostiene, por ejemplo, que "This close interrelatedness between humans and the non-human creation-the two cannot be separated from each other in plight and in salvation-most likely also continues in Rom 11 where Paul draws heavily on the OT and early Jewish concepts" (p. 286). (Agregar traducción de las citas en castellano para los lectores sin conocimiento de la lengua inglesa).

22 Harrington (2000) ha elucidado las respuestas que diversas tradiciones del AT han dado al impase generado por la irracionalidad del sufrimiento humano,y con él, el de las demás criaturas. Hasta la ruptura de la doctrina de la retribución, el sufrimiento humano fue expresión de un castigo justo con el propósito de corregir a las criaturas, es decir, el resultado del esfuerzo divino continuo por hacer de sus criaturas hijos auténticos (pp. 53-68).

23 El motivo de la gloria que las criaturas deben a su creador y padre retoma, de hecho, una idea importante de la carta: la gloria que se debe al Dios inmortal no se puede intercambiar por formas de idolatría $(1,23)$. 
una "gloriosa libertad". ${ }^{24}$ Acto seguido, el apóstol puntualiza, además, la consecuencia de tal filiación: la redención del cuerpo (v. 23). Si los motivos de la gloria y la filiación constituyen los hilos conductores del razonamiento paulino en esta sección, ¿por qué Pablo da un rodeo en los vv. 19-29 antes de llegar al clímax de la gloria manifestada y dada a sus hijos? ¿Acaso tiene que considerar alguna amenaza a su proyecto de gloria?

La tesis principal de la sección (sub-proposición) afirma que los padecimientos del tiempo presente no tienen comparación con la gloria que está por venir. Si bien los padecimientos se podrían considerar un obstáculo para la gloria, los verdaderos límites para ella se describen como frustración ( $\mu \alpha \tau \alpha$ ıó $\eta \varsigma)$ (v. 20),

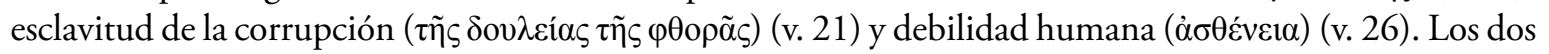
primeros se refieren a la creación, el tercero, a la humanidad; el segundo todavía podría atribuirse a la una o a la otra. La sucesión de estas amenazas abarca tanto la creación subhumana como la humana, y sugiere un movimiento que va de lo exterior, como la falta de un propósito, hacia lo interior, como la fragilidad.

¿Son estas, entonces, las amenazas contra la gloria destinada a los hijos de Dios? El vocabulario del gemir, de los dolores de parto, la imagen de los hijos que se esperan y la experiencia de la labilidad humana insinúan que el contexto bíblico que el apóstol tiene en mente es el de Gn 3,16. ${ }^{25}$ El designio divino trazado para Eva incluye generar hijos y dar a luz, la vida, con dolor. Esta expectativa de vida con dolor es fruto paradójico de la desobediencia. Así pues, junto con las amenazas enunciadas, el contexto bíblico remoto sugiere una amenaza, más grave aún, contra la gloria característica de los hijos, a saber, la desobediencia que frustra cualquier paternidad; pero que ya en el Génesis, desde los orígenes, ${ }^{26}$ se transforma, por querer divino, en fuente de vida.

El razonamiento paulino entreteje personajes (creación, nosotros, el Espíritu), adversarios (frustración, corrupción, labilidad) y un itinerario, a saber, el propósito divino para sus criaturas (la gloria y la filiación). La característica principal de esta argumentación, sin embargo, es la del clímax por medio de la negación; se niega para afirmar. Los sufrimientos no pueden, así como tampoco las amenazas pueden, frustrar la

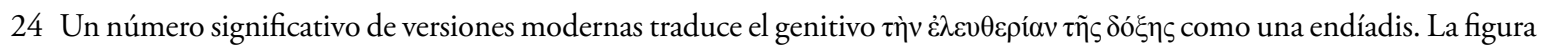
de "dos por uno" o "términos gemelos" aparece atestiguada en la carta y en el resto del NT (BDB $\$ 442.16$; Smyth, 1920, p. 3025; Mortara Garavelli, 1988, p. 219; Aletti et al., 2006, p. 95). En Rom 13,13 "comilonas glotonas" ( $\mu$ 门̀

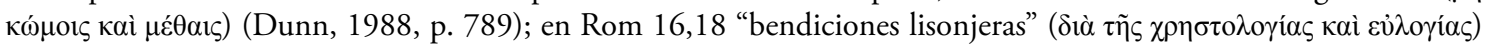
(Käsemann, 1974, p. 402; Wilckens, 1978, p. 142, n. 678). Véase otros ejemplos en Zerwick, 2011, $\$ 460$.

25 "Nor can there be any doubt that it was God himself who brought about the present condition of his own subhuman creation, doing so, as Gen 3:17 also emphasizes, 'because of you,' that is because of humanity's sin”. (Longenecker, 2016, pp. 722-723). (Agregar traducción de la cita al castellano para aquellos lectores sin conocimientos de la lengua inglesa).

26 Leese (2018) cree que Pablo, en Rom 8,18-30, interpreta la narración de la primera creación a la luz de Cristo: "Paul was aware of the first creation narrative and [...] he borrows from the language used there to teach his readers about the radical and new reality that has begun with the Christ event" (p. 121). (Ver comentario anterior). Él pasa por alto, sin embargo, el contexto temático sugerido por la narración inmediatamente posterior a la primera creación, a saber, el contexto de la desobediencia y la filiación. 
esperanza de la gloria. El crescendo del v. 30 corrobora el clímax: ${ }^{27}$ la voluntad divina (predestinación) se manifiesta como justificación de los creyentes, cuyo resultado es su glorificación. Así, el v. 30 concluye, no sólo el razonamiento de los vv. 18-30 mostrando cómo se alcanza la gloria, sino que también articula la noción de la justificación, demostrada en los capítulos anteriores, con la gloria que corresponde a los hijos de Dios. Algunos estudiosos han descubierto que este modo de razonar del apóstol tiene la forma

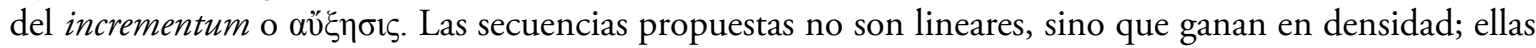
generan un espiral, en este caso, de esperanza. El incrementum señalado, en fin, se caracteriza por sugerir un desarrollo que va de los indicios más exteriores (creación / falta de propósito o dirección) a los más interiores (Espíritu / intención).

\section{La prosopopeya (пробшпопоиїа)}

La afirmación según la cual "la creación fue sometida a la frustración” forma parte de un razonamiento más amplio, cuyo propósito busca promover la esperanza en el designio divino y no la desilusión por su fracaso. Cabe preguntarse, sin embargo, si la imagen de la sujeción es pertinente o por lo menos proporcionada. Si no lo es, la argumentación del apóstol puede correr el riesgo de perder su capacidad persuasiva o, incluso, su coherencia. Si el objetivo de la argumentación paulina es demostrar que nada puede poner en riesgo la gloria que le espera a los hijos, ¿por qué no se afirma sin rodeos su ser habitados por el Espíritu, sin necesidad de comprometer la creación? Si la creación es buena e irresponsable del mal que la afecta, ¿cómo se justifica la amenaza desorbitante a la que se ve expuesta?

La interpretación de Crisóstomo a este pasaje puede ilustrar las herramientas retóricas puestas en juego por el apóstol para superar el impase generado por los padecimientos presentes de la creación y

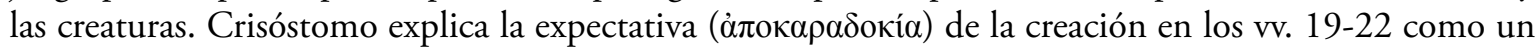
"aguardar intensificado". ${ }^{28} \mathrm{Su}$ interpretación del pasaje evidencia, todavía, un instrumento retórico mucho más importante para la comprensión de toda la sección, a saber, la personificación.

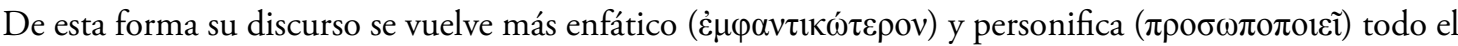

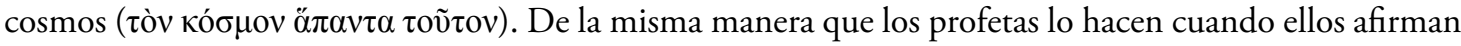
que los ríos baten sus palmas, o que las colinas y los montes saltan (Sal 114,6; Cant 2,8). Esta no es una forma fantasiosa de atribuirles poder, sino un instrumento para que nosotros podamos aprender la magnanimidad ( $\dot{\pi} \varepsilon \rho \beta \rho \lambda \eta \dot{)}$ de las bendiciones, tan grande como para alcanzar lo que aparece sin sentido.

27 "A quienes predestinó a estos los llamó. Y a quienes llamó, a estos los justificó. Y a quienes justificó a estos glorificó" (¿De dónde sale esta cita? Agregar referencia).. Pablo utiliza la figura retórica de la anadiplosis en otros pasajes de sus cartas (v.gr. Rom 5,4-5). Esta consiste en la repetición de términos por contacto; la repetición del último término de un sintagma al comienzo del siguiente. Los rétores antiguos la denominan reduplicatio. (Quintiliano, Inst., 9.3.29 [Russell, LCL]). Lausberg (1998) la clasifica como una variante especial de la geminatio (\$\$ 619-622). Véase también Mortara Garavelli, 1988, pp. 191-195.

28 Véase la traducción castellana de este esperar ( $\dot{\alpha} \pi \varepsilon \kappa \delta \varepsilon ́ \chi 0 \mu \alpha \imath)$ como “abrigar una esperanza” en Alonso Schöckel - Mateos (1975): "porque, [la humanidad] aun sometida al fracaso (no por su gusto, sino por aquel que la sometió), esta misma humanidad abriga una esperanza: que se verá liberada de la esclavitud a la decadencia, para alcanzar la libertad y la gloria de los hijos de Dios". (Agregar página). 
[...] Imitando a estos [los profetas] el apóstol personifica $(\pi \rho 0 \sigma \omega \pi \mathrm{o} \pi \mathrm{o} \varepsilon \tilde{i})$ a esta creación (

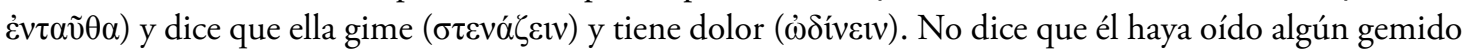

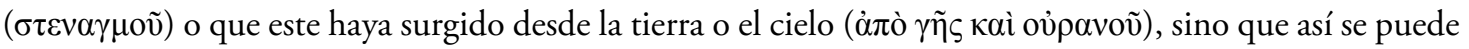
mostrar la magnanimidad ( $\tau \grave{v} v \dot{v} \pi \varepsilon \rho \beta 0 \lambda \eta ́ v)$ excelsa de las cosas buenas que están por venir y el deseo de transformación ( $\tau \tilde{\eta} \varsigma \alpha \dot{\alpha} \pi \alpha \lambda \lambda \alpha \gamma \tilde{\eta} \varsigma$ ) de las maldades que las sujetan. (Crisóstomo, In Espistulam ad Romanos, $60.529 .47-55.60) .^{29}$

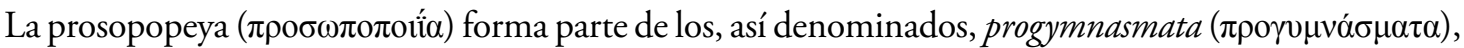
ejercicios preliminares para la formación de los rétores en la antigüedad. Esta aparece descrita ya en los ejercicios de Aelius Theón. ${ }^{30}$ Se define como la introducción de un personaje al cual se le atribuyen palabras adecuadas o al cual se le pueden aplicar de forma indisputable. En la carta a los Romanos, Pablo recurre a la prosopopeya en más de una ocasión. Él personifica, prácticamente, el pecado ( $\alpha \mu \alpha \rho \tau i ́ \alpha)$, siempre enunciado en singular; la misma observación vale para la ley (vó $\left.\mathrm{o}_{\mathrm{s}}\right)$. Ellos se describen como sujetos con

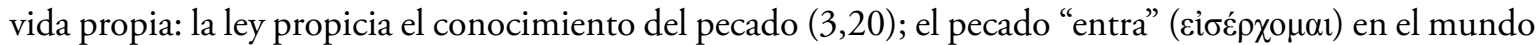

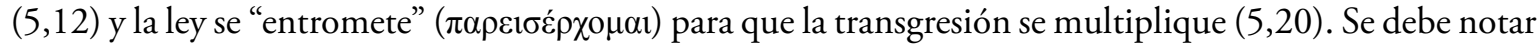
que Pablo, en sentido estricto, no "hace hablar" a ninguno de estos actores (pecado, ley, creación), sino que les atribuye acciones o actitudes características de personajes humanos. El efecto retórico que tal tipo de personificación persigue, como lo insinúa Crisóstomo, consiste en suscitar en el lector la experiencia

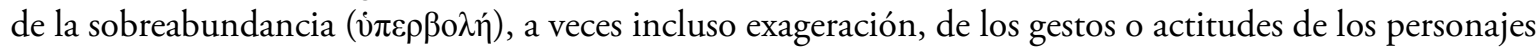
involucrados.

Pablo personifica la creación en Rom 8,19-22, atribuyéndole el aguardar ( $\dot{\pi} \pi \varepsilon \kappa \delta \varepsilon ́ \chi 0 \mu \alpha \iota)$, el gemir ( $\sigma \varepsilon v \alpha ́ \zeta \omega)$, el sentir dolor ( $\sigma v v \omega \delta i ́ v \omega)$. Él no "hace hablar" a la creación, pero caracteriza sus acciones de tal forma que hace de ella un personaje con actitudes propias. El hecho de estar sumisa o de haber sido sometida, pasivamente, se puede entender, entonces, como una forma de exageración retórica, útil a Pablo para subrayar —no la sumisión en sí misma—, sino su carácter irresponsable e indeseable.

\section{Consideraciones hermenéuticas}

¿Cuál es la creación que Pablo tiene en mente en Rom 8,19-22? ¿Humana o subhumana? Algunas versiones modernas, y no pocos estudiosos, la interpretan como la "humanidad". ${ }^{31}$ Esta creación no sería diferente de

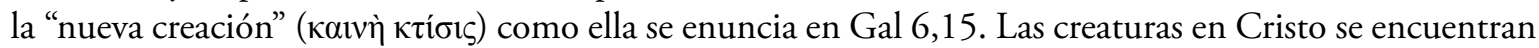
en un estado que supera las diferencias de género (hombre, mujer), étnico-religiosas (judío, griego) y sociales (libre, esclavo). La comprensión—escatológica—de la creación en la carta a los Romanos no sería

29 Schaff, 1974-75, p. 597.

30 Kennedy (2003) observa, sin embargo, que "Theon uses 'prosopopoeia' of any speech in character and is apparently unaware of the distinction between 'prosopopoeia,' 'ethopoeia,' and 'eidolopoeia' found in the later progymnastic treatises” (Agregar traducción de la cita al castellano) (p. 47). Véase también Gibson, 2008, pp. 355-425; Heath, 2002, pp. 129-160; Spengel, 1856, p. 10.

31 Véase supra, nota 25, el ejemplo de Alonso Schöckel - Mateos (1975). (Revisar numeración. La nota 25 no parece hacer referencia al mismo texto). 
sustancialmente diferente de la de Gálatas. ${ }^{32}$ Comentando la traducción de Crisóstomo, Schaff (1974-75) nota, sin embargo, que, si bien su interpretación de $\dot{\eta} \kappa \tau i ́ \sigma ı \varsigma$ parece muy general, ella se puede considerar correcta. Esta comprensión incluye en la creación a "todo el mundo", es decir, a las cosas sin sentido e inteligencia. ${ }^{33} \mathrm{Y}$ aunque la personificación de la creación pudiera sugerir al lector que el apóstol habla de la creación como si se tratara exclusivamente de la humanidad, el uso del verbo $\sigma v \sigma \tau \varepsilon v \alpha ́ \zeta \omega$ muestra que la creación, que se diferencia del "nosotros", los incluye porque gime igualmente, y se aúna, no sólo a la esperanza de la liberación, sino también a la de la gloria que le aguarda a los hijos.

¿Cuál es el contexto bíblico que explica las afirmaciones de Rom 8,19-22? ¿Cuál es su consecuencia para la reflexión teológica sobre el medio ambiente"? Leese (2018) considera Gen 1-2 el contexto más adecuado para interpretar los vv. 19-22. Él cree, además, que la teología de la creación de Ireneo refleja, en primer lugar, la hermenéutica intertextual de Pablo (que posiciona a la humanidad dentro del espectro de la oíкоvouía divina), y, en segundo lugar, la cristologización de la narrativa de la primera creación (192-193). Él sostiene, por lo mismo, que la ecoteología puede encontrar, en este modelo, intuiciones para su desarrollo. La ecología puede llegar a—o debería—ser un filtro hermenéutico (hermeneutical lens) para reapropiarse las escrituras de forma creativa (p. 194). Tal perspectiva puede, además, "corregir" la tradición occidental dominante, según la cual, la redención está relacionada con la caída de Adán y Eva en Gen 3 (Watson, 2010, p.130). Según Leese (2018), la teología paulina de la creación debería seguir los derroteros trazados por Ireneo, quien interpreta la creación, primero a partir de Gen 1-2, y sólo, de forma secundaria, a partir de Gen 3 (p. 196).

El énfasis dado a Gen 1-2 por el análisis intertextual de Rom 8,19-22 subraya la dimensión cósmica del sometimiento de la creación. La redención que se aguarda, por lo mismo, es de todo el cosmos. Esta interpretación ha sido ampliamente utilizada por algunos entusiastas del diálogo entre ecología y Biblia. Moo (2008) afirma, por ejemplo, que "Romans 8.19-22 is quite possibly the text most frequently cited by those seeking to employ Christian Scripture for an environmental agenda" (p. 74). Stenschke (2017) comparte la misma observación y subraya uno de los motivos centrales del proyecto "Uses of the Bible in Evironmental Ethics" de la Universidad de Exeter, el cual definió Rom 8,19-23 como un "environmental mantra” (p. 262).$^{34}$ Esta interpretación, sin embargo, minusvalora la contradicción posible: ¿por qué un mundo creado bueno-como el de Gen 1-2—-tendría que ser sometido al fracaso? ¿ Solo para ver redimida a la humanidad al final?

La lectura cuidadosa de Rom 8,18-30 muestra, en primer lugar, que el incrementum en el razonamiento del apóstol confirma la técnica de la inclusión. Pablo deja claro que las creaturas están incluidas en la creación; las amenazas a la gloria divina afectan, por igual, a la creación humana y subhumana. En segundo lugar, el vocabulario del gemir (compuestos $\sigma \tau \varepsilon v-)$, unido al del sufrimiento ( $\sigma v v \omega \delta i ́ v \omega)$, sugiere que el

32 "The 'new creation' in its fulness belongs to the future, but to those in Christ it is already realized through the Spirit". (Agregar traducción al castellano) (Bruce, 1982, p. 273).

33 Schaff (1974-75) se decanta, en realidad, por la interpretación subhumana de la creación: "It is more likely that the apostle has in mind distinctively the irrational creation. [...]. Nature is subject to "vanity"-i.e. the law of decay and death, and is poetically spoken of as awaiting the revelation of the sons of God in the hope of sharing in it" (p. 597). (Ver nota anterior)

34 Véase también Byrne, 2010, p. 262, en un volumen dedicado solo a la Biblia y la cuestión ambiental: Horrell et al. (2010). 
contexto más plausible para la interpretación de Rom 8,19-22 es el de Gen 3: la respuesta divina a la desobediencia humana. Se trata de una respuesta paradójica, porque ella describe la acción divina como sujeción y su efecto como opresión y dolor que generan vida. Esta forma de proceder confirma que la paradoja y la personificación son los instrumentos adecuados para hacer digerible la iniciativa divina, a pesar de la labilidad humana (Aletti, 2012, p. 35 y p. 213): la gloria para sus hijos. Gieniusz (1999) sintetiza así nuestra comprensión del incrementum en el razonamiento paulino y sus consecuencias:

In all of them, 8:20 included, Paul's way of speaking on the whole could be considered a kind of exaggeration ( $\alpha$ ̋̋ $\eta \sigma ı \varsigma)$ which he shares with other biblical authors (Ex 8:15.32; 9,34; 1 Sam 6:6; God's hardening Pharaoh's heart: Ex 4:21; 7:3.4.13; 9:12.15-17), but which is not without precise theological sense. In fact, if he shadows the human fault, and consequently emphasizes divine "causality", he does so not in order to deny the human fault and so to make God the one, and even less the only one, responsible of evil but to indicate that even the actions of humans (the Fall in case of Rom 8:20), are previewed and integrated in God's plan and cannot oppose effectively its definitive accomplishment (p. 159).

\section{Conclusiones}

¿Quién sometió a la creación y con qué objetivo? Varios estudiosos de la carta consideran que esta sujeción es confusa. ${ }^{35}$ Wilckens (1978), por ejemplo, afirma que el v. 20 es oscuro. Aunque gran parte de la

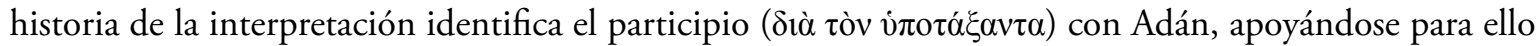
en 4Esd 7,11, Wilckens (1978) cree que no tiene sentido que los pecados de Adán o su culpa recaigan sobre la creación. Él también considera dudoso que el participio másacusativo de persona se identifique con Dios (p. 154) ${ }^{36}$ Igualmente, Bultmann (1953) concluye de forma inconcluyente: "Eine Eindeutige Entscheidung jedoch scheint mir nicht möglich" (p. 230). A diferencia de estos y otros autores, nuestro análisis muestra que la ambigüedad introducida por el participio (

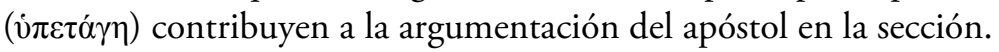

El razonamiento del apóstol, en Rom 8,18-30, se construye de forma progresiva e inclusiva. Pablo utiliza la prosopopeya ( $\pi \rho \circ \sigma \omega \pi$ oлotía), de forma creativa, para mostrar que creación humana y subhumana están igualmente amenazadas. El peligro del fracaso consiste en no alcanzar-o perder-el propósito dispuesto por Dios para unos y otros: la gloria de los hijos de Dios. La comprensión del incrementum

35 "Es ist darum sehr warscheinlich, dass Paul hier nicht von Adam, sondern wiederum von Gott spricht und nun hervorheben will dass es nicht die Schuld der Schöpfung, sondern alleinige Entscheidung Gottes war, die Schöpfung

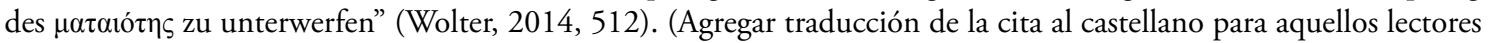
que no conocen la lengua alemana).

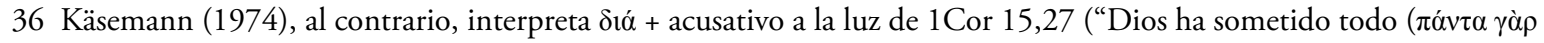

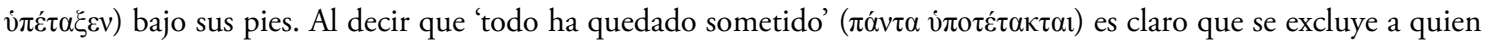

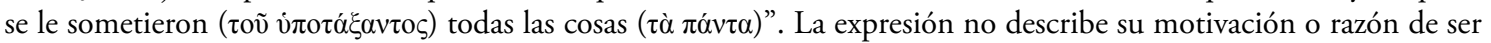
(Beweggrund), sino su creador o autor (Urheber) (p. 227). Ni la creación, ni el ser humano se pueden considerar culpables de la sujeción de la creación. Dios la ha "sometido". Käsemann (1974) subraya así la voz pasiva del verbo vं

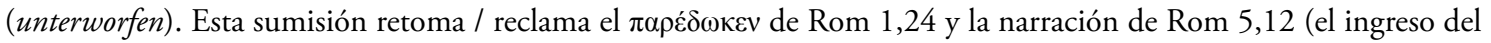
pecado en el mundo). 


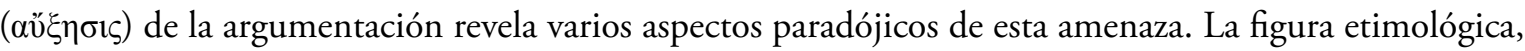
es decir, el crescendo creado mediante los compuestos $\sigma \tau \varepsilon v-$, muestra que la sujeción de la creación está aunada a un pujar-presión, dolor y opresión—que genera vida. La progresión indica no solo el carácter inaudito de la sujeción, sino la esperanza que ella misma suscita. El incrementum indica, además, que el Espíritu se asocia a este pujar. En consonancia con la línea argumentativa de los vv. 12-17, el Espíritu divino capacita el entendimiento ( $\left.\varphi \rho v_{\eta} \mu \alpha\right)$ humano para comprender que ningún peligro, ni siquiera la desobediencia de los mismos hijos, puede frustrar el proyecto divino. Este desarrollo sugiere, en fin, que el pasaje se puede interpretar exculpando a la creación y a las criaturas; su sujeción, involuntaria, demuestra que ellas no enfrentan solas el dolor y la opresión, sino que Dios puja con ellas.

\section{Referencias}

Aletti, J-N., Gilbert, M., Ska, J-L. y de Vulpillières, S. (2006). Lessico ragionato dell'esegesi biblica. Le parole, gli approcci, gli autori. Brescia: Queriniana.

Aletti, J-N. (2012). New Approaches for Interpreting the Letters of Saint Paul: Collected Essays; Rhetoric, Soteriology, Christology and Ecclesiology. Rome: Pontifical Biblical Institute.

Byrne, B. (2010). "An Ecological Reading of Rom 8.19-22: Possibilities and Hesitations". En D. Horrell et al. (Eds.), Ecological Hermeneutics: Biblical Historical and Theological Perspectives (pp. 83-93). London - New York, NY: T\&T Clark.

Bruce, F.F. (1982). The Epistle of Paul to the Galatians: A Commentary on the Greek Text. Grand Rapids, MI: Eerdmans.

Bultmann. (1953). Theologie des Neuen Testaments. Tübingen: Mohr Siebeck.

Dunn, J.D.G. (1988). Romans 1-8. WBC 38A. Dallas, TX: Word Books.

Fernández Galiano, M. (1986). Esquilo: Tragedias. Madrid: Gredos.

Gibson, C.A. (2008). Libanius' Progymnasmata: Model Exercises in Greek Prose Composition and Rhetoric. SBL. Writings from the Greco-Roman World 27. Atlanta, GA: SBL.

Gieniusz, A. (1999). Romans 8:18-30: "Suffering Does Not Thwart Future Glory”. Atlanta, GA: Scholars Press.

Granados. J.M. (2012). "Reconciliation in Col 1, 20: A Case of Polyptoton". En F. Bianchini y S. Romanello. (Eds.), Non mi vergogno del vangelo, potenza di Dio (pp. 153-170). Roma: Pontifical Biblical Institute.

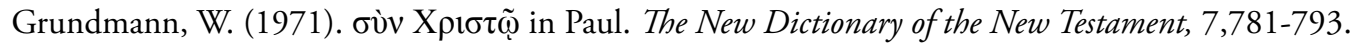

Harrington, D.J. (2000). Why do We Suffer? A Scriptural Approach to the Human Condition. Franklin, WI: Sheed $\&$ Ward.

Heath, M. (2002). Theon and the History of Progymnasmata. Greek Roman and Byzantine Studies, volumen 43(2), $129-160$.

Jewett, R. y Kotansky, R.D. (2007). Romans: A Commentary (E.J. Epp, Ed.). Minneapolis, MN: Fortress Press.

Käsemann, E. (1974). An die Römer. . Tübingen: Mohr Siebeck.

Kennedy, G.A. (2003). Progymnasmata: Greek Textbooks of Prose Composition and Rhetoric. Leiden: Brill. 
Lausberg, H. (1998). Handbook of Literary Rhetoric: A Foundation for Literary Study. Leiden: Brill.

Leese, J.J.J. (2018). Christ, Creation, and the Cosmic Goal of Redemption: A Study of Pauline Creation Theology as Read by Irenaeus and Applied to Ecotheology. London: T \& T Clark.

Longenecker, R.N. (2016). The Epistle to the Romans: A Commentary on the Greek Text. Grand Rapids, MI: Eerdmans.

Moo, J. (2008). Romans 8.19-22 and Isaiah's Cosmic Covenant. New Testament Studies, 54(1), 74-89. https://doi. org/10.1017/S0028688508000052

Mortara Garavelli. B. (1988). Manuale di Retorica. Milano: Bompiani.

Quintiliano.(2002). Institutio oratoria. The Orator's Education, Volume IV. (D.A. Russell,Ed.). Cambridge, MA: Harvard University Press. DOI: 10.4159/DLCL.quintilian-orators_education.2002. https://doi.org/10.4159/ DLCL.quintilian-orators_education.2002

Schaff, P. (Ed.) (1974-75). A Select Library of the Nicene and Post-Nicene Fathers of the Christian Church. Vol. 11: Saint Chrysostom: Homilies on the Acts of the Apostles and the Epistle to the Romans. Grand Rapids, MI: Eerdmans.

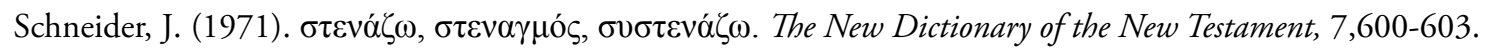

Stenschke, C. (2017). Human and Non-Human Creation and Its Redemption in Paul's Letter to the Romans. Neotestamentica, 51(2), 261-289. https://doi.org/10.1353/neo.2017.0014

Smyth, H.W.S. (1920). Greek Grammar for Colleges. New York, NY: American Book Company.

Spengel von, L. (1856). Rhetores Graeci ex recognitione. Vol III. Lipsiae: Teubner.

Watson, F. (2010). "In the Beginning: Irenaeus, Creation and Environment". En D. Horrell et al. (Eds.), Ecological Hermeneutics: Biblical Historical and Theological Perspectives (pp. 127-139). London: T\&T Clark.

Wilckens, U. (1978). Der Brief an die Römer: (Röm 6-11). Zürich: Benziger.

Wolter, M. (2014). Der Brief an die Römer. Neukirchen-Vluyn: Patmos.

Zerwick, M. (2011). Biblical Greek Illustrated by Examples. (J. Smith, Traductor). Rome: Pontifical Biblical Institute. 